\title{
TM4SF5-mediated protein-protein networks and tumorigenic roles
}

\author{
Jung Weon Lee* \\ Department of Pharmacy, Research Institute of Pharmaceutical Sciences, Tumor Microenvironment Global Core Research Center, \\ Medicinal Bioconvergence Research Center, College of Pharmacy, Seoul National University, Seoul 151-742, Korea
}

Transmembrane $4 \mathrm{~L}$ six family member 5 (TM4SF5), as a membrane glycoprotein with 4 transmembrane domains, is similar to the tetraspanins in terms of membrane topology and plays important roles in tumorigenesis and tumor metastasis. Especially, TM4SF5 appears to form a massive protein-protein complex consisting of diverse membrane proteins and/or receptors in addition to cytosolic signaling molecules to regulate their signaling activities during the pathological processes. TM4SF5 is shown to interact with integrins $\alpha 2, \alpha 5$, and $\beta 1$, EGFR, IL6R, CD151, focal adhesion kinase (FAK), and c-Src. This review focuses on the significance of the interactions with regards to TM4SF5-positive tumorigenesis and metastasis. [BMB Reports 2014; 47(9): 483-487]

\section{INTRODUCTION}

TM4SF5, transmembrane 4 L6 family member 5, has a similar membrane topology to the tetraspanins (TM4SFs). They commonly have four transmembrane domains, two short cytosolic $\mathrm{N}$ - and C-terminal tails, and two extracellular loops (SEL and LEL, short extracellular loop 1 and long extracellular loop 2, respectively) $(1,2)$. It is suggested that the tetraspanins form massive protein-protein complexes on the cell membrane with tetraspanins in hemophilic or heterophilic manners and other membrane receptors including growth factor receptors and integrins $(2,3)$. These protein complexes including tetraspanins can regulate cell adhesion, proliferation, and migration at a compartmentalized membrane (micro)domain called tetraspanin-enriched microdomain (TEM) (4-6).

TM4SF5 is highly expressed in liver (7) and prostate cancers (8). It appears to form TM4SF5-enriched microdomains while regulating cellular functions via formation of protein com-

*Corresponding author. Tel: +82-2-880-2495; Fax: +82-2-8721795; E-mail: jwl@snu.ac.kr

http://dx.doi.org/10.5483/BMBRep.2014.47.9.146

Received 11 July 2014

Keywords: Cytokine receptors, EGFR, Fibrosis, Integrins, Metastasis, Tetraspanin, TM4SF5 plexes with tetraspanin CD151, integrins, growth factor receptors, and cytokine receptors (Fig. 1). In this review, TM4SF5 that interacts and collaborates with other membrane proteins during its pro-tumorigenic roles will be explained.

\section{INTERACTION BETWEEN TM4SF5 AND MEMBRANE RECEPTORS}

There are three different levels of interactions that form a massive protein complex at TEM, where the tetraspanins seems to play functions as an architectural guide $(9,10)$; (i) the primary interaction level accounts for direct interactions between tetraspanins and their binding partners, defined as those that remain associated with a given tetraspanin under conditions disrupting the tetraspanin-tetraspanin interactions; (ii) the secondary interaction is when the primary interaction complexes are linked into larger masses via the tendency of tetraspanins to interact with each other; and (iii) the tertiary interactions are defined as the tetraspanin interactions that are disrupted in detergents of Brij96/97 and triton X-100, but are retained in less hydrophobic detergents of Brij58, Brij 99, Brij 35, and CHAPS, leading to incomplete solubilizations of tetraspanins and membrane fractions in sucrose gradients (4).

\section{TM4SF5 as a component of tetraspanin-enriched microdomain (TEM)}

As a membrane glycoprotein, similar to the tetraspanins in terms of membrane topology, TM4SF5 is also thought to be localized at a TM4SF5-enriched microdomain (i.e., $\mathrm{T}_{5} \mathrm{EM}$ ). Via complex formations with diverse categories of membrane protein or receptors and even intracellular signaling molecules, TM4SF5 appears to play critical roles in tumorigenic and metastatic processes. TM4SF5 binds with integrins, growth factor receptors, tetraspanins, and even cytokine receptors, in addition to non-receptor tyrosine kinases of focal adhesion kinase (FAK) and c-Src.

\section{TM4SF5 interaction with integrins}

TM4SF5 interacts with integrins $\alpha 2, \alpha 5$, or $\beta 1$, during co-immunoprecipitation approaches (3). The interaction of TM4SF5 with either $\alpha 2$ or $\alpha 5$ integrin chain was differentially studied in terms of detergent within lysis buffer and interfacing 


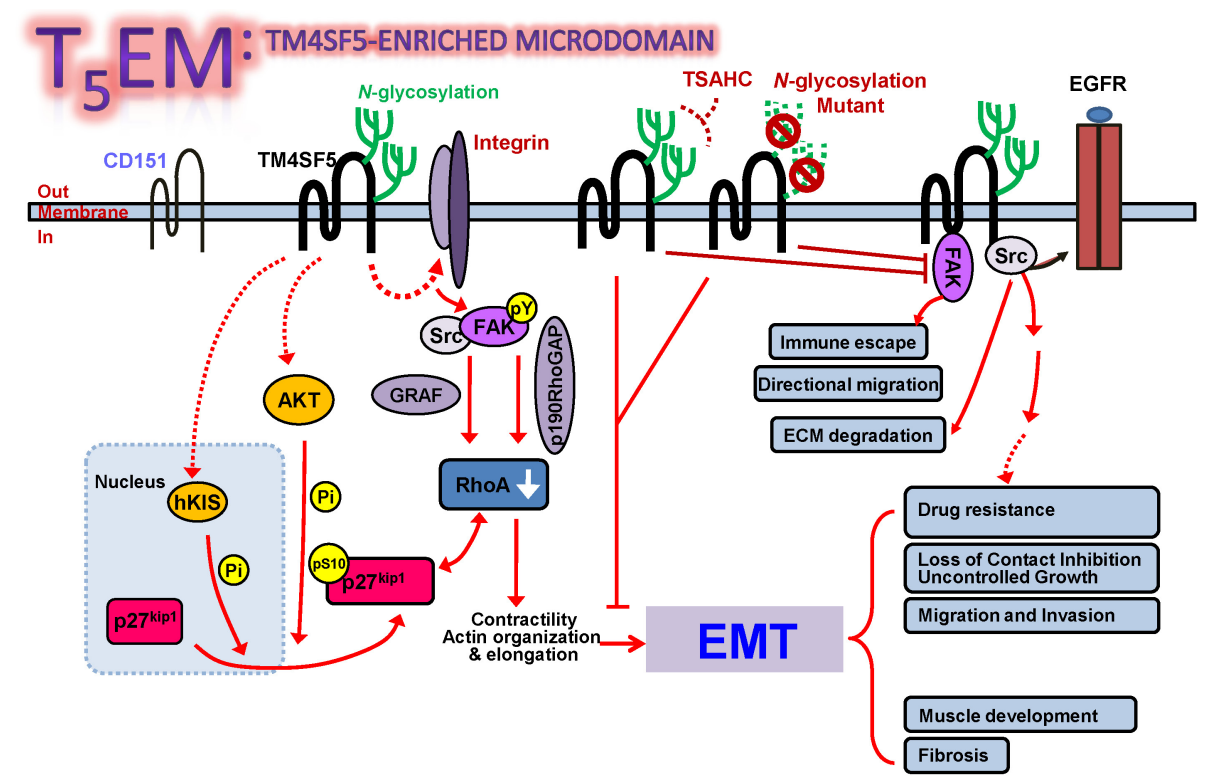

Fig. 1. The working model for the TM4SF5-mediated roles in tumorigenesis and metastasis. regions of the molecules. TM4SF5 interacts with the cytoplasmic tail of integrin $\alpha 5$ in Brij58-solubilized SNU449 hepatocyte extracts (11), whereas it associates with integrin $\alpha 2 \beta 1$ through but not with $\alpha 3, \alpha 5$, or $\alpha 6$ in SDS-solubilized hepatocyte extracts (12). This interaction with integrin $\alpha 2 \beta 1$ depends on the $\mathrm{N}$-glycosylation status in the LEL (long extracellular loop 2 or the extracellular loop 2, EC2) or structural intactness of the LEL of TM4SF5 (12). Disturbing the association between TM4SF5 and integrin $\alpha 2$ using a peptide consisting of a part of the LEL or EC2 or anti-TM4SF5 reagent [4'-(p-toluenesulfonylamido)-4-hydroxychalcone, TSAHC, (13)] that seems to interfere with its $\mathrm{N}$-glycosylation results in recoveries in spreading on and migration toward collagen I (12) or blocks the TM4SF5-mediated multilayer growth or loss of contact inhibition (13), respectively. These studies suggest that TM4SF5 is also capable of modulating the signaling properties of integrins through physical associations, playing roles in cell adhesion and migration through actin reorganization. In addition to tumorigenic roles, functional linkage between TM4SF5 and integrin $\alpha 5$ during muscle development of zebrafish has been observed; suppression of TM4SF5 in zebrafish during development impairs the expression and localization of integrin $\alpha 5$ and upstream and downstream effectors including fibronectin, FAK, vinculin, and actin, eventually leading to abnormal muscle development (14).

TM4SF5 interactions with epidermal growth factor receptor The interaction between TM4SF5 and integrin $\alpha 2$ also occurs in fibroblasts; TM4SF5 regulates dynamics of focal adhesion formation and actin organization depending on its interaction with $\alpha 2$ and the interaction is negatively modulated by serum treatment (15). This observation suggests that the interaction between TM4SF5 and integrin $\alpha 2$ is blocked when growth factor receptors are activated and the activated EGFR may become associated with TM4SF5. The interaction between TM4SF5 and EGFR is observed by a co-immunoprecipitation approach using NSCLC expressing TM4SF5, where the interaction is positively correlated with the gefitinib resistance even without EGFR mutation (e.g., T790M) following TM4SF5 overexpression (16).

Interestingly enough, the EGFR signaling system is involved in TGF 31 -mediated Smads activation leading to TM4SF5 induction (17). TM4SF5 is importantly enhanced during fibrosis development in $\mathrm{CCl}_{4}$-adminstrated mouse, and pharmacological inhibition of EGFR activity blocks TM4SF5 induction (18). Given that more than $80 \%$ of liver fibrosis and cirrhosis leads to HCC $(19,20)$, the importance of TM4SF5 in fibrosis development can similarly account for the HCC development. Further, we found that endogenous EGFR was bound to ectopically overexpressed TM4SF5 in hepatic cancer cells and their colocalization was observed at the front tips of migratory cells (HJ Kim and JW Lee, unpublished observation). Therefore, the interaction between EGFR and TM4SF5 can play roles in migration, in addition to tumorigenesis and drug resistance.

\section{TM4SF5 interactions with cytokine receptors}

TM4SF5 expression involves TGF $\beta 1$-mediated Smad activation, Smad4-mediated EGF secretion, and EGFR activation on plasma membrane (17). TM4SF5 is enhanced in fibrotic livers of mice administrated with $\mathrm{CCl} 4$, and treatment of anti-TM4SF5 reagent TSAHC results in blockade of the fibrosis (18). Since liver fibrosis development is well-known to involve multifunctional cytokine TGF $\beta 1$ (21), live fibrosis 
development in $\mathrm{CCl} 4$-adminstrated mice could involve TGF $\beta 1$-mediated TM4SF5 expression.

In addition to TGF $\beta 1$, diverse cytokines can be related to fibrosis and tumorigenesis $(22,23)$. Cytokines consist of tumor microenvironment and thereby the functions of tumor cells can be influenced by the cytokines. When TM4SF5-null and -expressing epithelial cells were analyzed for cytokine expression using an antibody array approach, normal hepatocytes lacking for TM4Sf5 expressed less interleukin 6 (IL6) than normal hepatocytes with enforced TM4SF5 expression. This correlates with a less effective homeostatic migration capacity of TM4SF5-null cells than TM4SF5-positive normal hepatocytes. However, TM4SF5-null cancer cells express more IL6 than TM4SF5-positive cancer cells, so that TM4SF5-positive cancer cells can have less influence on TM4SF5-dependent FAK activity by the IL6/STAT3-mediated immunological activity, consequently leading to TM4SF5-enhanced migration and invasion during metastasis (24). During this TM4SF5-mediated immune-escape action TM4SF5 appears to interact with IL6R through their extracellular domains, since $\mathrm{N}$-glycosylation mutant TM4SF5 impaired the interaction, but a deletion of the cytosolic domain of IL6R did not affect the binding (24). Therefore, TM4SF5 functions appear to correlate with the roles of cytokines or cytokine receptors during development of fibrosis and tumorigenesis.

\section{TM4SF5 interaction with other tetraspanins}

Being similar to the tetraspanins, TM4SF5 can localize at its own TEM ( $\left.\mathrm{T}_{5} \mathrm{EM}\right)$, where other tetraspanins can also localize together. When other tetraspanin expressions are analyzed between TM4SF5-null and chronically-TGF $\beta 1$-treated (thereby TM4SF5-expressing) hepatocytes, CD151 is increased in TM4SF5-positive cells whereas CD9, CD63, CD82, CD105, and CD117 are not (25). Immunostaining of the colocalization between TM4SF5 and CD151 is not always occurring; a certain population of TM4SF5 on the plasma membrane is co-localized with certain CD151, suggesting migration enhancements through individual or collaborative functions (25). TM4SF5 is upstream of CD151, since TM4SF5 regulates CD151 mRNA and protein levels and overwhelm the CD151-mediated migration, but conversely CD151 does not change the levels of TM4SF5 (25). Meanwhile, in case of CD63, a tumor suppressor, TM4SF5 expression results in internalization of CD63 into late lysosomal membranes, positively costained with LAMP2, so that the CD63's tumor suppressive functions on the cell surface can be minimized (25). Therefore, similar to immune-escape by lower expression of IL6 in TM4SF5-positive cancer cells, it appears very likely that TM4SF5-expression can lead to aggressive tumorigenic roles overwhelming the possible actions by tumor suppressors.

\section{INTERACTION BETWEEN TM4SF5 AND INTRACELLULAR SIGNALING MOLECULES}

Since extracellular ligands for most tetraspanins are not yet known and interactions with other secreted proteins or membrane proteins/receptors on the same or opposing cells can regulate intracellular signaling emanated by the binding proteins, it may be possible to speculate that binding to other molecules can be an initiation process of tetraspanin-mediated signaling. Although the tetraspanins are suggested not to be responsible for cell adhesion and spreading processes, TM4SF5 is shown to directly interact with FAK and c-Src in a cell-ECM adhesion-dependent manner and thereby leads to enhanced migration and invasive ECM degradation, respectively, through their activations.

\section{Interaction between TM4SF5 and FAK for migration}

TM4SF5 expression itself results in activated FAK even without any stimulation (7). Further, expression of GFP-tagged TM4SF5 in hepatocytes leads to localizations at membrane edges including the lammelipodia and the filopodia of migratory cells (17). Therefore, TM4SF5 at the leading edges of migratory cells can direct cell migrations. TM4SF5 interaction with FAK occurs through the intracellular loop domain of TM4SF5 and the F1 lobe of the FAK FERM domain, so that the interaction can lead to an opening of inhibitory intramolecular interaction between the FAK FERM (F2 lobe) and the kinase (C-lobe centered on Phe596) domain (26) for an autophosphorylation of Tyr397 (27).

TM4SF5 can activate FAK presumably at an earlier adhesion time. When epithelial cells are replated onto poly-L lysines that are not based on integrin-ECM adhesion or on ECM following a preincubation of the cells with functional-blocking antibody against integrin $\alpha 1$, the FAK Tyr397 phosphorylation is clearly higher than that of suspended cells. This indicates that presumably prior to earlier integrin clustering during cell adhesion processes TM4SF5 alone interacts and causes structural changes in FAK for its autophosphorylation, but as time passes for the cell-ECM adhesion, integrins cluster and get involved in FAK activation via a synergistic collaboration with TM4SF5 (27). TM4SF5-dependent FAK activity is required for chemotactic and haptotactic migration, invasive ECM degradation, and eventually lung metastasis. Furthermore, the TM4SF5 mutant not binding FAK or treatment of anti-TM4SF5 reagent TSAHC results in blockade of the TM4SF5/FAK interaction-mediated effects.

\section{Interaction between TM4SF5 and c-Src for invasion}

In addition to FAK, TM4SF5 directly interacts with c-Src through the C-terminal tail of TM4SF5 and the SHI kinase domain of c-Src [(28) and Song HE and Lee JW, unpublished observations]. Interestingly enough, TM4SF5 more efficiently recruits inactive c-Src, of which Tyr527 phosphorylation is abundant or Tyr416 phosphorylation is minimal (28). However, 
TM4SF5 expression results in c-Src activation, compared with TM4SF5-null cells (7). The interaction-mediated c-Src activity is required for invasive protrusion formation and ECM degradation capacities and involves EGFR phosphorylation at Tyr845 residue. Such TM4SF5/c-Src interaction-mediated c-Src activity can lead to activation of cortactin for enhanced actin branching or reorganization around lammelipodia (28). Therefore, these observations support that the TM4SF5 C-terminus-mediated modulation of c-Src (family kinase) activity can be targeted to block metastasis of TM4SF5-positive tumors. Understanding modulation of the TM4SF5-mediated c-Src activity at the molecular levels and looking for tools to modulate the mechanisms would be clinically beneficial.

\section{CONCLUSION}

As a membrane glycoprotein, TM4SF5 appears to be highly expressed in pathological conditions including fibrotic and tumor cells or tissues. Although its ligand has not been identified, its interactions with diverse tumorigenic membrane receptors or intracellular signaling molecules lead to aggressive intracellular signaling activities for cell morphology, migration, and invasion. Although the interactions with different molecules may have differential affinities or natures for the binding interfaces, targeting the protein-protein interaction may be a promising strategy to block the TM4SF5-mediated tumorigenesis and metastasis.

\section{REFERENCES}

1. Veenbergen, S. and van Spriel, A. B. (2011) Tetraspanins in the immune response against cancer. Immunol. Lett. 138, 129-136.

2. Detchokul, S., Williams, E. D., Parker, M. W. and Frauman, A. G. (2013) Tetraspanins as regulators of the tumour microenvironment: implications for metastasis and therapeutic strategies. Br. J. Pharmacol. doi: 10.1111/bph. 12260.

3. Lee, S. A., Park, K. H. and Lee, J. W. (2011) Modulation of signaling between TM4SF5 and integrins in tumor microenvironment. Front. Biosci. 16, 1752-1758.

4. Hemler, M. E. (2003) Tetraspanin proteins mediate cellular penetration, invasion, and fusion events and define a novel type of membrane microdomain. Annu. Rev. Cell Dev. Biol. 19, 397-422.

5. Hemler, M. E. (2005) Tetraspanin functions and associated microdomains. Nat. Rev. Mol. Cell Biol. 6, 801-811.

6. Yanez-Mo, M., Barreiro, O., Gordon-Alonso, M., Sala-Valdes, M. and Sanchez-Madrid, F. (2009) Tetraspanin-enriched microdomains: a functional unit in cell plasma membranes. Trends. Cell Biol. 19, 434-446.

7. Lee, S. A., Lee, S. Y., Cho, I. H., Oh, M. A., Kang, E. S., Kim, Y. B., Seo, W. D., Choi, S., Nam, J. O., TamamoriAdachi, M., Kitajima, S., Ye, S. K., Kim, S., Hwang, Y. J., Kim, I. S., Park, K. H. and Lee, J. W. (2008) Tetraspanin TM4SF5 mediates loss of contact inhibition through epithelial-mesenchymal transition in human hepatocar- cinoma. J. Clin. Invest. 118, 1354-1366.

8. Muller-Pillasch, F., Wallrapp, C., Lacher, U., Friess, H., Buchler, M., Adler, G. and Gress, T. M. (1998) Identification of a new tumour-associated antigen TM4SF5 and its expression in human cancer. Gene 208, 25-30.

9. Berditchevski, F. and Odintsova, E. (2007) Tetraspanins as regulators of protein trafficking. Traffic 8, 89-96.

10. Charrin, S., le Naour, F., Silvie, O., Milhiet, P. E., Boucheix, C. and Rubinstein, E. (2009) Lateral organization of membrane proteins: tetraspanins spin their web. Biochem. J. 420, 133-154.

11. Choi, S., Lee, S. A., Kwak, T. K., Kim, H. J., Lee, M. J., Ye, S. K., Kim, S. H., Kim, S. and Lee, J. W. (2009) Cooperation between integrin $\alpha 5$ and tetraspan TM4SF5 regulates VEGF-mediated angiogenic activity. Blood 113, 1845-1855.

12. Lee, S. A., Kim, Y. M., Kwak, T. K., Kim, H. J., Kim, S., Kim, S. H., Park, K. H., Cho, M. and Lee, J. W. (2009) The extracellular loop 2 of TM4SF5 inhibits integrin $\alpha 2$ on hepatocytes under collagen type I environment. Carcinogenesis 30, $1872-1879$.

13. Lee, S. A., Ryu, H. W., Kim, Y. M., Choi, S., Lee, M. J., Kwak, T. K., Kim, H. J., Cho, M., Park, K. H. and Lee, J. W. (2009) Blockade of four-transmembrane L6 family member 5 (TM4SF5)-mediated tumorigenicity in hepatocytes by a synthetic chalcone derivative. Hepatology 49, 1316-1325.

14. Choi, Y. J., Kim, H. H., Kim, J. G., Kim, H. J., Kang, M., Lee, M. S., Ryu, J., Song, H. E., Nam, S. H., Lee, D., Kim, K. W. and Lee, J. W. (2014) TM4SF5 suppression disturbs integrin $\alpha 5$-related signaling and muscle development in zebrafish. Biochem. J. 462, 89-101.

15. Lee, S. Y., Kim, Y. T., Lee, M. S., Kim, Y. B., Chung, E., Kim, S. and Lee, J. W. (2006) Focal adhesion and actin organization by a cross-talk of TM4SF5 with integrin $\alpha 2$ are regulated by serum treatment. Exp. Cell Res. 312, 2983-2999.

16. Lee, M. S., Kim, H. P., Kim, T. Y. and Lee, J. W. (2012) Gefitinib resistance of cancer cells correlated with TM4SF5-mediated epithelial-mesenchymal transition. Biochim. Biophys. Acta. 1823, 514-523.

17. Kang, M., Choi, S., Jeong, S. J., Lee, S. A., Kwak, T. K., Kim, H., Jung, O., Lee, M. S., Ko, Y., Ryu, J., Choi, Y. J., Jeong, D., Lee, H. J., Ye, S. K., Kim, S. H. and Lee, J. W. (2012) Cross-talk between TGFb1 and EGFR signalling pathways induces TM4SF5 expression and epithelial-mesenchymal transition. Biochem. J. 443, 691-700.

18. Kang, M., Jeong, S. J., Park, S. Y., Lee, H. J., Kim, H. J., Park, K. H., Ye, S. K., Kim, S. H. and Lee, J. W. (2012) Antagonistic regulation of transmembrane 4 L6 family member 5 attenuates fibrotic phenotypes in $\mathrm{CCl}_{4}$-treated mice. FEBS. J. 279, 625-635.

19. Sangiovanni, A., Del Ninno, E., Fasani, P., De Fazio, C., Ronchi, G., Romeo, R., Morabito, A., De Franchis, R. and Colombo, M. (2004) Increased survival of cirrhotic patients with a hepatocellular carcinoma detected during surveillance. Gastroenterology 126, 1005-1014.

20. Severi, T., van Malenstein, H., Verslype, C. and van Pelt, J. F. (2010) Tumor initiation and progression in hepatocellular carcinoma: risk factors, classification, and therapeutic targets. Acta. Pharmacol. Sin. 31, 1409-1420. 
21. Ghosh, A. K., Quaggin, S. E. and Vaughan, D. E. (2013) Molecular basis of organ fibrosis: potential therapeutic approaches. Exp. Biol. Med (Maywood). 238, 461-481.

22. Meindl-Beinker, N. M., Matsuzaki, K. and Dooley, S. (2012) TGF-beta signaling in onset and progression of hepatocellular carcinoma. Dig. Dis. 30, 514-523.

23. Mantovani, A., Savino, B., Locati, M., Zammataro, L. Allavena, P. and Bonecchi, R. (2010) The chemokine system in cancer biology and therapy. Cytokine. Growth. Factor. Rev. 21, 27-39.

24. Ryu, J., Kang, M., Lee, M. S., Kim, H. J., Nam, S. H., Song, H. E., Lee, D. and Lee, J. W. (2014) Cross-talk between the TM4SF5/FAK and the IL6/STAT3 pathways renders immune escape of human liver cancer cells. Mol. Cell Biol. 34, 2946-2960.

25. Kang, M., Ryu, J., Lee, D., Lee, M. S., Kim, H. J., Nam, S. H., Song, H. E., Choi, J., Lee, G. H., Kim, T. Y., Lee, H., Kim, S. J., Ye, S. K., Kim, S. and Lee, J. W. (2014) Correlations between Transmembrane 4 L6 family mem- ber 5 (TM4SF5), CD151, and CD63 in liver fibrotic phenotypes and hepatic migration and invasive capacities. PLOS ONE 9, e102817. doi: 10.1371/journal.pone. 0102817.

26. Lietha, D., Cai, X., Ceccarelli, D. F., Li, Y., Schaller, M. D. and Eck, M. J. (2007) Structural basis for the autoinhibition of focal adhesion kinase. Cell 129, 1177-1187.

27. Jung, O., Choi, S., Jang, S. B., Lee, S. A., Lim, S. T., Choi, Y. J., Kim, H. J., Kim, D. H., Kwak, T. K., Kim, H., Kang, M., Lee, M. S., Park, S. Y., Ryu, J., Jeong, D., Cheong, H. K., Park, K. H., Lee, B. J., Schlaepfer, D. D. and Lee, J. W. (2012) Tetraspan TM4SF5-dependent direct activation of FAK and metastatic potential of hepatocarcinoma cells. J. Cell Sci. 125, 5960-5973.

28. Jung, O., Choi, Y. J., Kwak, T. K., Kang, M., Lee, M. S., Ryu, J., Kim, H. J. and Lee, J. W. (2013) The COOH-terminus of TM4SF5 in hepatoma cell lines regulates c-Src to form invasive protrusions via EGFR Tyr845 phosphorylation. Biochim. Biophys. Acta. 1833, 629-642. 